\title{
INNOVACIÓN EN LA ENSEÑANZA UNIVERSITARIA
}

\author{
MIGUEL A. ZABALZA \\ Universidad de Santiago de Compostela
}

\begin{abstract}
RESUMEN: Innovar no es sólo hacer cosas distintas sino hacer cosas mejores. Y mantener los cambios hasta tanto se haya podido consolidar la nueva cultura que los cambios (cuando son cambios y no meros sucedáneos) conllevan necesariamente.
\end{abstract}

Este artículo trata de algunos dilemas teóricos y prácticos que están presentes en la innovación de las prácticas docentes en la universidad. Innovar en la docencia se ha convertido en una exigencia institucional sometida a muchas presiones y no pocas contradicciones. Por eso resulta importante tomar ser consciente de las posibilidades y limitaciones reales que enmarcan el espacio de la innovación y mejora de la docencia en la universidad. En el marco de ese propósito analítico se van repasando distintos modelos de innovación en la universidad en función de su contenido, de su modalidad y del nivel de impacto. Finalmente se entra a considerar el aspecto crucial de cualquier intento de innovación, pero más si cabe en la universidad: la formación del profesorado universitario. Varios factores influyen de forma clara y constatada en la efectividad de los actuales programas de formación pedagógica del profesorado universitario: la mentalidad del profesorado (incluyendo en ella tanto sus creencias pedagógicas como sus actitudes hacia el desempeño docente); su disponibilidad de tiempo (o la densidad de su agenda); la existencia de referentes y ejemplos de buenas prácticas; las propias estrategias de formación que se sigan. El artículo concluye con el análisis de dos retos básicos y urgentes que tiene abiertos en este momento la innovación docente: el reto de los métodos docentes y el reto de la documentación, intercambio de experiencias y reajuste de las prácticas.

ABSTRACT: Innovating is not only to do different things, but to do better things. And to keep the changes until the consolidation of the new culture which the changes (when they are changes and not mere substitutes) necessarily convey.

This article deals with some of the theoretical and practical dilemmas which are present in the innovation of the teaching methods in the university. Innovation in teaching has become an institutional demand, subjet to many pressures and no fewer contradictions. That is why it is important to be aware of the real possibilities and limitations which set the place for innovation and 
improvement of teaching in the university. In the framework of that analytic purpose, different innovation models in the university are checked according to their content, their modality and their impact level. Finally, the crucial aspect of any innovation attempt is considered, especially in the university: the training of its teaching staff. Several factors influence in a clear and confirmed way in the effectiveness of the current pedagogical training programs for the university teaching staff: the teacher's mentality (including their pedagogical beliefs and attitudes towards their teaching performance); their time availability (or the density of their agendas); the existence of referents and examples of good teaching practice; their own teaching strategies. The article concludes with the analysis of two basic and urgent challenges which teaching innovation is currently working on: the challenge of teaching methods and the challenge of the documentation, exchange of experiences and readjustment of teaching practice

PALABRAS CLAVE: Innovación docente, docencia universitaria, formación del profesorado universitario, didáctica universitaria.

KEYWORDS: Teaching innovation, university teaching, university teacher training, university didactic.

\section{INTRODUCCIÓN : el nuevo escenario universitario}

Suele decirse, y más adelante insistiré en ello, que no todo cambio conlleva mejora de la cosa que se cambia. Pero parece obvio que, por sentido común, si uno cambia algo lo hace para mejorar. En eso estamos en la universidad. Enfrascados en un torbellino de cambios que, presumiblemente, han de llevarnos a conseguir una universidad mejor. Mejorar la calidad de la enseñanza en las Universidades se ha convertido, así, en una de las prioridades más socorridas en la actualidad. No conozco ni un sólo programa electoral de los nuevos equipos que han ido ocupando los rectorados de las Universidades en la época post-LOU que no mencione esa meta en un lugar preferente de su oferta programática. Tampoco conozco ninguna universidad en la que no se hayan puesto en marcha todo un torbellino de iniciativas en torno al proceso de convergencia europea. Es bien cierto que las interpretaciones que se han hecho de ese propósito de mejora son muy diversas pero, en su conjunto, dan a entender que los nuevos responsables universitarios están prestando una atención a la docencia universitaria como nunca antes se había hecho.

Desde luego, no es una preocupación nueva. $\mathrm{Ni}$ es, por supuesto, resultado de la aparición de nuevas leyes universitarias o del nuevo contexto de homologación de los estudios. Quiero entenderlo, más bien, como la progresiva sedimentación de una progresiva valoración de la docencia que ha ido madurando en estos últimos años. Hasta hace poco la docencia, en sí misma, no constituía un asunto relevante para la universidad como institución. Salvo en situaciones excepcionales, no se hablaba de ello en los órganos académicos (salvo de cuestiones formales referidas a programas, horarios, etc.), no se tomaban decisiones al respecto, no se valoraban las prácticas docentes ni se establecían planes serios para mejorarlas. En aras de la "libertad de cátedra", la calidad de la docencia quedaba en manos de los profesores individuales o, como mucho, bajo la tutela de los Departamentos. El resultado habitual ha sido que cada uno de nosotros "hacía de su capa un sayo" en sus clases. Las aulas y labo- 
ratorios se han convertido, en la tradición universitaria, en escenarios "opacos" y poco transparentes, espacios "privados" donde, por lo general, cada profesor impone sus propias reglas y dinámicas de funcionamiento.

Pese a que la situación en la actualidad dista mucho de ser halagüeña, afortunadamente las cosas van cambiando. Lentamente, eso sí, y con no pocas dificultades. Si comparamos la Universidad de hoy con la de hace una década, los cambios han sido sustantivos y, en general, podríamos decir que se ha incrementado de manera notable la calidad de las enseñanzas universitarias. Y no solamente porque han mejorado las infraestructuras y se han modernizado los sistemas de gestión sino, sobre todo, porque ha ido cambiando la mentalidad sobre el sentido de la formación y el papel de la universidad y de la docencia en el desarrollo personal y profesional de los estudiantes.

Algunas "certezas" (quizás hablar de "convicciones" resulte más apropiado) que afectan a la docencia han ido sedimentándose, como digo, en estos últimos tiempos. Entre ellas podríamos resaltar las siguientes:

1.- Que la docencia en sí misma es un componente importante en la formación de nuestros estudiantes. Una buena docencia marca diferencias entre unos centros universitarios y otros. Lo que los universitarios aprenden depende, ciertamente, de su interés, esfuerzo y capacidades pero depende también de que hayan tenido buenos o malos docentes, mejores o peores recursos, de que les hayan ofrecido unas u otras oportunidades de aprendizaje.

2.- Que la docencia pertenece a un tipo de actuación con características propias y distintas de los otros cometidos que el profesorado universitario debe asumir. Enseñar es distinto de investigar y es igualmente distinto de llevar a cabo tareas de gestión, de extensión cultural o de implementar otros proyectos profesionales (informes, auditorias, asesorías, etc.).

3.- Que ser capaz de realizar una buena enseñanza no es cuestión de mucha práctica. La práctica ayuda, sin duda, pero por sí sola resulta insuficiente. Sólo cuando la práctica va acompañada de formación y de revisión (algunos prefieren hablar de "reflexión") es cuando se hace posible el conocer cada vez más a fondo los entresijos del aprendizaje de los alumnos y el ajustar mejor nuestro trabajo docente a las condiciones y propósitos de la formación.

4.- Que, al final, como cualquier otra profesión, la docencia constituye un espacio de competencias profesionales. Esas competencias, también en la enseñanza universitaria, están compuestas por conocimientos (sobre los contenidos que se enseñan y sobre los propios procesos de enseñar y aprender), por ciertas habilidades específicas (de comunicación, de manejo de recursos didácticos, de gestión de métodos, de evaluación, etc.) y por un conjunto de actitudes propias de los formadores (disponibilidad, empatía, rigor intelectual, etc.).

Desde luego no se trata, en absoluto, de batallas ganadas. Muchas de esas "convicciones" están todavía en proceso de debate y asentamiento. Pero lo importante es que, ahora sí, ya están ahí. Forman parte del debate académico, se van rompiendo estereotipos y certezas previas que las negaban. Resulta obvio que, pese a tratarse de cuestiones que a un pedagogo le pueden parecer "de cajón", no todos nuestros colegas de las distintas especialidades universitarias (cuya opinión de la Pedagogía, por otra parte, es más bien pobre) están de acuerdo en asumirlas. Algunas de ellas con- 
tradicen ciertos "principios" que han configurado el "sentir" tradicional de la mayoría de los docentes universitarios:

- Que a enseñar se aprende enseñando.

- Que para ser un buen profesor universitario basta con ser un buen investigador.

- Que "aprender" es una tarea que depende exclusivamente del alumno; los profesores deben dedicarse a enseñar (explicar) los temas. Si aprenden o no es cosa que los alumnos deben resolver por su cuenta y bajo su responsabilidad.

- Que una universidad es de calidad no tanto por las clases que se imparten cuanto por los recursos de que se dispone: buenos laboratorios, buenas bibliotecas, suficientes recursos en nuevas tecnologías, etc.

No será fácil superar estas viejas ideas que, pese a las innumerables evidencias en contra, algunos defienden como principios incontestables. Quizás por ello, en los últimos tiempos ha ido instalándose, también, una cierta pose de escepticismo: es imposible, se dice, intentar transformar la universidad, a lo menos en lo que a la enseñanza se refiere. Demasiados intereses, demasiado individualismo, demasiado peso de la tradición y de viejos vicios en la forma de actuar. Atribuyen a un Ministro portugués de Educación aquello de que "las Universidades no se transforman, simplemente hay que crear otras nuevas". Pero lo interesante, como señalaba, es que incluso en este contexto de incertidumbre y visiones contrapuestas, y pese a tener la tradición en contra, las Universidades (sus Equipos Rectorales, sus Facultades, muchos Departamentos y bastantes profesores y profesoras individuales) se han ido comprometiendo estos últimos años en iniciativas interesantísimas de formación para la docencia, de programas de innovación, de planes estratégicos de mejora de la docencia y de sus resultados.

En esas estamos y a ello pretenden contribuir las numerosas experiencias que, en el ámbito de la innovación y en otros, se han ido poniendo en marcha en casi todas las universidades. Tanto los políticos como los responsables de las Universidades han entendido bien que mejorar la calidad de la docencia es un propósito que requiere de la sinergia institucional y de la implicación de los diversos sectores de la comunidad universitaria; que se trata de un proceso lento que, además, ha de pasar necesariamente por la formación del profesorado. Siendo verdad que la "calidad de la enseñanza universitaria" requiere de la existencia de buenas bibliotecas y suficientes recursos didácticos y tecnológicos, todo ello sirve de poco si el profesorado no se siente comprometido y con motivación, ganas y condiciones para iniciar procesos de cambios. Este es el gran reto actual de nuestras universidades.

Éste es un proceso que no ha surgido espontáneamente. Las presiones externas (en este momento, las derivadas de la Declaración de Bolonia y de todo el proceso de convergencia hacia el Espacio Europeo de Educación Superior) están jugando un papel fundamental. También lo están haciendo las nuevas condiciones demográficas que han reducido drásticamente el número de alumnos poniendo en riesgo la subsistencia de ciertas carreras y alterando el equilibrio financiero de las instituciones universitarias en su conjunto. Las universidades se ven obligadas a competir en la captación de estudiantes y para ello han de mostrar su "mejor imagen" como instituciones de formación. De hecho, el nuevo papel que han ido adquiriendo los estudiantes ha tenido, desde luego, una fuerte incidencia en la génesis de este nuevo 
escenario. A medida que los estudiantes han ido teniendo más voz institucional se ha ido haciendo evidente que, al menos desde su perspectiva, hay buenos y malos profesores. Valoración que tiene poco que ver con la experiencia de dicho profesorado o con su competencia investigadora o con su relieve público. Simplemente son competentes o incompetentes como profesores aunque puedan ser estupendos investigadores o buenos gestores. Y esa "imagen" de la docencia que los estudiantes transmiten acaba actuando a favor o en detrimento de la "atracción" que las universidades ejercen sobre los futuros universitarios.

Es en ese contexto en el que estamos ahora. Como fruto del nuevo escenario en el que se mueve la universidad, la docencia ha adquirido un nuevo sentido y ha pasado a ser un elemento relevante de la actuación de las universidades. De ahí que las instituciones universitarias estén Ilamadas a generar condiciones organizativas, financieras y culturales que estimulen iniciativas de mejora de la enseñanza universitaria.

\section{LA INNOVACIÓN EN LA UNIVERSIDAD}

Cuando se revisan los programas de innovación de las universidades que los tienen establecidos y los proyectos que suelen presentarse a dichas convocatorias, uno puede quedar bastante perplejo ante la heterogeneidad de planteamientos con que se aborda la innovación. Por eso quizás venga bien una pequeña reflexión sobre qué significa (y qué no significa) innovar en la universidad. Es bien cierto que nadie puede intentar sentar cátedra al respecto y en absoluto es ésa mi intención, pero me parece necesario señalar algunos principios que rigen los procesos de innovación.

\section{¿Qué no es innovar?}

Aunque exigiría de mayores matizaciones, podríamos comenzar diciendo que una cosa es cambiar y otra bien distinta en innovar. Innovar no es sólo hacer cosas distintas sino hacer cosas mejores. Innovar no es estar cambiando constantemente (por aquello de identificar innovación con cambio) sino introducir variaciones como resultado de procesos de evaluación y ajuste de lo que se estaba haciendo. La cuestión es introducir procesos innovadores que vayan asentando prácticas que supongan una mejora de la calidad de lo que se está haciendo. Esta idea del afianzamiento y consolidación de los procesos iniciados suele exigir un cierto tiempo (que las cosas se vayan sedimentando, que tanto las personas participantes como las instituciones vayan "aprendiendo" a gestionar y sacar el máximo partido a las innovaciones) y entra en contradicción con la obsesión por cambiar por estar haciendo siempre cosas distintas.

Uno de los principales enemigos de la innovación auténtica son las innovaciones forzadas (por la vía formal, burocrática o administrativa). Cuando una institución se ve forzada a cambiar (a establecer un servicio nuevo, a generar documentos o planes de actuación distintos a los anteriores, etc.) lo que suele acontecer es que la innovación se produce pero sólo formalmente y sin capacidad de impacto sobre las prácticas reales. A una demanda burocrática se responde con una acción también burocrática. 
Algo de eso ha venido sucediendo con los sucesivos documentos institucionales que tanto los departamentos como los profesores hemos tenido que ir generando estos últimos años en relación a los programas de nuestras asignaturas. Si el departamento exige el programa de la asignatura los profesores, aunque sea a regañadientes lo hacen y entregan. Si las Facultades o Escuelas exigen que los departamentos entreguen la programación de las materias que se les encomiendan, lo hacen. Pero ni en uno ni en otro caso, esa acción acaba mejorando la docencia (o nuestra propia reelaboración de la materia que impartimos): es una respuesta burocrática a una demanda del mismo signo.

Ni qué decir tiene que la innovación no es un mecanismo para obtener fondos suplementarios. No está mal conseguir esos fondos, desde luego. Muchas innovaciones los requieren y no saldrían adelante sin ellos. Otras veces, los fondos suplementarios vienen bien como estimulación añadida para que el profesorado se implique en iniciativas que le van a exigir esfuerzo y consumir tiempo. Pero cuando los proyectos de innovación se instrumentalizan en exceso (el objetivo está más en la obtención de fondos que en el contenido en sí de la innovación) las expectativas con respecto a dichos proyectos no pueden ser elevadas, dado que el objetivo no es mejorar la docencia sino la cuenta de resultados (o el equipamiento, o los recursos).

Algo parecido, en cuanto a la instrumentación, puede acontecer si, como parece justo, la innovación acaba convirtiéndose en un mérito valorable en la carrera docente del profesorado universitario. Que se valore el esfuerzo innovador de cada profesor no debería llevarnos a que las innovaciones propuestas estén más orientadas a la contabilidad de méritos que a la mejora lograda en la docencia y en el aprendizaje de los estudiantes.

La innovación no es un salvoconducto. La calidad de la innovación va a depender, justamente, de la calidad de la propuesta innovadora no del hecho en sí de que se trate de una innovación. La tendencia habitual en la universidad a distinguir entre "métodos tradicionales" y "métodos innovadores"; entre "profesores innovadores" y "profesores clásicos (o retrógrados)"; entre programas o instituciones "convencionales" y otros "innovadores" no hace sino confundir el sentido y orientación de la innovación. La confusión se hace aún más grave si acabamos identificando "innovador" con bueno y "no innovador" con malo. Al hablar de Innovación (al menos de innovación educativa) no podemos sustituir lo sustantivo (formación, profesor/a, programa) por lo adjetivo (innovador/a). Lo importante es ser buen profesor, o buen programa o buena institución. De esta manera lo sustantivo quedaría como sustantivo y el atributo de "innovador" como una cualidad adjetiva que matiza y cualifica lo fundamental. Lo adjetivo ("innovadores") no debe suplantar o sobreponerse a lo sustantivo ("profesores"). La cuestión o la exigencia básica que cabe plantear a los profesores es que sean buenos "profesores". Si además son innovadores mejor. Pero no conviene confundir una cosa con la otra. Seguramente hay muy buenos profesores poco proclives a participar en experiencias de innovación (en los formatos habituales que las innovaciones presentan: cambios sobre las formas habituales de actuar). Y el hecho de que no podamos incluirlos en la categoría de profesores innovadores no justifica en absoluto el que deban ser incluidos, por exclusión, en la de "malos profesores".

Un buen profesor es el que hace su trabajo con responsabilidad, dedicación y conocimiento de causa. Esto es lo sustantivo de un buen profesional. En ese sentido, la innovación es algo añadido. Algo diferente a la sustancia del quehacer profesio- 
nal. Eso no quiere decir que no sea importante, que lo es, sino que conviene que no mezclemos las cosas. Todos conocemos buenos profesores que son más reacios que otros a implicarse en un proyecto concreto o que no manifiestan una disponibilidad fácil a participar en las diversas iniciativas de cambio que periódicamente van surgiendo en los centros o son impuestas desde la Administración Educativa.

Es frecuente encontrarse con profesores que son muy renuentes al cambio. Sobre todo cuando no ven claro que lo nuevo que se propone vaya a mejorar lo que ya había, o cuando entienden que la mejora previsible resulta muy descompensada con respecto al esfuerzo suplementario que requiere la nueva iniciativa. Suelen preferir seguir haciendo las cosas como las venían haciendo, a incorporar cambios. Prefieren hacer las cosas bien a hacerlas distintas. Y, en principio, parece una postura respetable.

A veces, aunque nos parece interesante la idea que nos proponen y nos encantaría participar en ella nos damos cuenta de que siendo realistas no estamos en ese momento en condiciones de participar por todo el conjunto de razones antes señaladas. Algunas personas esto lo aceptan con normalidad y lo dicen abiertamente. Otras, sienten reparos en confesarlo (en parte por el sentido moralizador al que antes aludí : si lo confesaran parecerían "malos profesores/as") y lo que hacen es disfrazarlo de doctrina (crean argumentos, virtualmente científicos o, si no, basados en su experiencia para descalificar cualquier intento de innovación). En todo caso, la innovación, en cuanto implicación personal en experiencias concretas de cambio, es algo que dependerá de nuestro mejor criterio profesional. Y también, sin duda, de otros aspectos secundarios pero que adquieren una importancia básica en el ámbito relacional : simpatía con los promotores de la experiencia, contexto personal-familiar en que nos encontremos en ese momento, factores coyunturales diversos, etc.

Por supuesto, este intento de clarificación no debe ser tenido como una defensa del inmovilismo. Ni tampoco como una desestimación de la innovación. Ni una cosa ni otra entran en mi planteamiento. Pero un cierto relativismo con respecto a la idea de innovación creo que resulta muy importante.

La innovación no debería una fuente permanente de discordias. A veces resulta frustrante para quienes hacen propuestas de innovación sentir un escaso respaldo de los colegas y pueden tener la tentación de plantearlo como una cuestión de "buenos" y "malos" profesores, una batalla entre profesores motivados y otros poca interesados en la docencia. Hacer de la innovación un campo de batalla no suele ser bueno para la propia innovación: no sólo no se conseguirá que los más fríos se adhieran a ella sino que se les pondrá en contra. De todas maneras, debe quedar claro que una visión "relajada" y "constructiva" de la innovación tiene como único sentido la evitación de que la "innovación" se convierta en un factor de ruptura institucional y de incomunicación entre colegas. Algunos expertos han defendido ese planteamiento (la innovación como "denuncia" y como "ruptura" cuasi revolucionaria) que personalmente no comparto. Es cierto que toda innovación trae consigo conflictos puesto que cualquier cambio supone romper con estructuras anteriores para crear otras nuevas y eso nunca es algo fácil. Pero ya tenemos bastante con afrontar el conflicto y los problemas sustantivos que generan las innovaciones como para suponer que, además de eso, podamos también cargar con la tensión que genera la descalificación y ruptura con los colegas menos dispuestos a colaborar. Pero esa virtual "neutralidad" no debe ser confundida con el "laisez-ferismo", con que todo dé igual e incluso con la defensa de la pasividad y desinterés. 


\section{¿Qué SÍ es innovar?}

En contraposición con lo señalado en la cara negativa de la innovación, lo primero que podemos decir en esta revisión en positivo de la innovación es que innovar es introducir cambios justificados (poniendo más énfasis en lo de justificados que en lo de cambio sin más). La calidad del cambio dependerá de lo valioso que sea el cambio en sí mismo y de la justificación que tenga (por qué razón se introdujo y en base a qué criterios se espera que mejoren las cosas).

Por otra parte, innovar en docencia es aplicar tres condiciones importantes en todo ejercicio profesional: apertura, actualización, mejora. La apertura está unida a la flexibilidad, capacidad de adaptación, etc. No se trata de cambiar algo rígido por otra cosa igualmente rígida. Las innovaciones rígidas, demasiado terminadas y elaboradas potencian la "fidelidad" más que el "compromiso activo" y aunque pueden mejorar los dispositivos formativos y sus resultados no tienen buen pronóstico sobre todo porque no generan una cultura innovadora en el centro escolar (que se basa más en el desarrollo de una actitud favorable al cambio y a la introducción de reajustes cuando sea proceso). La actualización tiene que ver con la puesta al día. Cuando se introducen nuevos modelos y/o formas de actuación docente no se trata de improvisar o de inventarse algo, sino de poner al día los sistemas de enseñanza incorporando los nuevos conocimientos y recursos disponibles. En ese sentido la actualización se opone al estancamiento, a la arqueología didáctica, a la ocurrencia coyuntural, al desconocimiento del progreso disciplinar y profesional vinculado al propio campo de actuación. Es frecuente que las innovaciones se planteen como una opción alternativa sin más ( como hacer algo distinto) pero al margen de un estudio previo de por dónde van las cosas, qué se sabe de ese tema, qué otras experiencias se han hecho y cuáles han sido sus resultados, etc. Es decir, sin una voluntad explícita de actualización de los procesos que se pretende mejorar. La mejora es un compromiso de toda innovación : perece un contra-sentido pensar que se introduce algo diferente para empeorar las cosas. Algunas, sin embargo, podrían describirse de ese modo. No son pocos los que critican muchas innovaciones como "retrocesos" y pérdida de la calidad.

Una tercera consideración positiva a la innovación es la que hace referencia a la importancia de la documentación y evaluación. Innovar es tomar decisiones vinculadas desde su inicio a procesos de evaluación. Todo cambio debería ir acompañado de sistemas de documentación, supervisión y evaluación (que permitieran incorporar los reajustes precisos sobre el propio cambio).Todo cambio debería ser evaluado al final para analizar su efectividad y su pertinencia. Y para decidir, igualmente las condiciones en que procedería mantenerlo, si así se decidiera. Estamos demasiado habituados a introducir innovaciones sin saber por qué las introducimos (falta de datos iniciales) y a abandonarlas sin saber tampoco por qué (falta de evaluación posterior).

También habría que recoger algunas condiciones prácticas de las innovaciones en la universidad. Una de ellas es la condición de practicidad. En este sentido, las innovaciones deben ser por un lado viables (posibilidad efectiva de ser llevada a cabo) y, por otro, prácticas (que no se quede en meras palabras o posibilidades sino que dé lugar a resultados tangibles). Es difícil convencer a nadie y garantizar que la propuesta de cambio prospere si no se justifica la practicidad del cambio, su viabilidad efectiva contando con los recursos disponibles. El gran problema de muchas innova- 
ciones educativas es que se trata de propuestas prácticamente utópicas o cuya puesta en marcha efectiva implicaría un equipamiento (de personal, conocimientos y recursos) del que no se dispone. Por otra parte es importante que la propuesta innovadora no se quede en palabras (ideas, valores, planteamientos genéricos, etc.) sino que incorpore componentes tangibles, algo que se pueda tocar, de lo que se pueda discutir, que se pueda presentar como resultado o consecuencia "real" del proceso de innovación: un producto, una grabación, una publicación o un informe final. En fin, algo objetivo que poder presentar. Ese algo práctico ejerce un fuerte efecto motivador sobre los participantes en la experiencia innovadora. Al ser algo objetivo y público permite además compartir la experiencia con los demás, someterla a debate, reajustarla si fuera preciso, etc.

Otra condición importante de las innovaciones es la formalización del proyecto, aunque sea un nivel de formalización"light". Aunque algunos profesores minusvaloran esta cuestión puesto que la consideran puramente formal y secundaria ("Io importante es tener la idea, dicen, no tenerla escrita") las cosas no funcionan así. Cualquier iniciativa de innovación debe contar con un proyecto escrito donde se describa y pormenorice (hasta donde sea posible) el previsible desarrollo de la misma. Varias razones avalan esta pretensión:

a) La existencia del documento escrito obliga a "pensar por adelantado" todo el proyecto. Es decir uno tiene que tener una visión general (aunque en esta fase sea una visión esquemática y abierta a cambios durante su desarrollo) de todo el proceso. Cuantos hayan tenido la experiencia de poner en marcha una innovación saben de sobra qué diferente es tener una intuición o idea brillante y ponerse después a configurar el proyecto completo. Esta segunda tarea es mucho más compleja, exige una disciplina muy fuerte, te obliga a concretar las ideas, a establecer una secuencia de fases, a buscar un equilibrio entre propósitos y recursos disponibles, etc.

b) La existencia del documento convierte la iniciativa en algo público y que puede ser compartido. Lo podemos presentar a los demás, lo podemos someter a sus críticas o propuestas de mejora (y también lo podemos defender de ellas), lo podemos reajustar en función de las sugerencias recibidas, etc.

c) La existencia del documento escrito permite confirmar tanto la coherencia como la viabilidad del intento. Justamente por que se trata de algo "pensado" y de algo "público" el documento escrito desvela la propia estructura interna de la iniciativa (y ahí se podrá ver si las actividades propuestas y la forma de llevarlas a cabo es coherente con los propósitos de la innovación) y su pronóstico (si los medios y recursos con los que se cuenta, si los apoyos financieros, institucionales y de personal hacen previsible que la actuación pueda llevarse efectivamente a cabo).

d) La existencia de un documento escrito constituye una especie de "compromiso formal" sobre el desarrollo de la actividad propuesta. Todos sabemos que cuando hay unos papeles de por medio las cosas son diferentes. En la medida en que en el proyecto se hayan señalado las metas a alcanzar, los dispositivos a poner en marcha para lograrlo, los recursos que se van a emplear, etc. eso queda ahí y queda por escrito, lo cual le otorga a la iniciativa un mayor contenido de "credibilidad" y firmeza. Cuando la iniciativa que se pone en marcha compromete a varias instancias o diversos agentes (individuales o en grupo), la clarificación escrita de los compromisos que cada uno de los participantes asume es una condición fundamental para que las cosas vayan bien. El plan no puede quedar en un acuerdo verbal porque luego las palabras se las lleva el viento y podemos (o pueden) quedarnos colgados en cualquier momento. 
El que se insista en la necesidad de un documento escrito no supone una postura cerrada y rígida con respecto a la naturaleza del documento. No es que tenga que ser algo absolutamente minucioso y en el que se prevea cualquier movimiento. Por eso decía en el encabezamiento que ese documento puede ser tan básico y light como se quiera. El nivel de precisión dependerá de las exigencias del guión: de quiénes vayan a participar en el acuerdo (y en función de ello de lo necesario que sea atar bien los cabos antes de iniciar la experiencia), del tipo de iniciativa o innovación de que se trate (hay algunos temas o ámbitos que exigen un mayor nivel de precisión que otros, en algunos basta con señalar las líneas maestras del proyecto), de lo elaborada que esté la propuesta (las iniciativas en una fase inicial no pueden concretar tanto lo que va a ser su desarrollo futuro pues no se tiene experiencia suficiente como para poder prever su evolución).

Finalmente, las innovaciones, las buenas innovaciones, deben evolucionar en el sentido de ser incorporadas al currículum formativo de la Facultad o Escuela en la que se hayan puesto en marcha. Aunque al hablar de enseñanza parece como que estamos ya metidos en el currículum, eso no es así exactamente. Se hacen muchas cosas en la enseñanza, entre ellas muchas innovaciones, que no logran integrarse en el currículum formativo que ese centro ofrece. Las innovaciones pueden plantearse con sentido en sí mismas, como experiencias estancas y autoreferidas. Se trata de acciones aisladas, puntuales. En ese caso su valor va a depender de las características de la propia innovación, pero en general su aportación suele resultar bastante marginal y acaba no afectando a la mejora de la educación en su conjunto. Eso es lo que suele pasar con muchas iniciativas interesantes de profesores aislados: proponen actividades dentro o fuera del horario lectivo pero que no forman parte del programa formativo (visitas, participación en trabajos prácticos, intercambios, metodologías didácticas novedosas, etc.). Son actividades que están metidas en el horario e incluso en la planificación anual, se cuenta con ellas al organizar el curso, pero no están metidas en el currículum, no forman parte de los dispositivos que el centro organiza para alcanzar los objetivos formativos que se habían planteado para esa etapa. Son cosas interesantes pero sueltas. Las buenas innovaciones están destinadas a ser incorporadas al proyecto educativo de esa titulación: deberían aparecer (tras algunos años de experimentación como iniciativas personales) como parte de lo oferta formativa que hace ese centro. Lo mismo que se hacen sesiones de laboratorio porque lo piden los programas, o sesiones de trabajo físico porque la formación incluye ese ámbito, también las innovaciones se deberían poder incorporar al programa porque constituyen parte del proceso global a través del cual se trata de alcanzar los propósitos formativos establecidos para todos los estudiantes.

En definitiva, estamos ante una innovación cuando se pretende llevar a cabo un proceso de cambio bien fundamentado. Cambios viables y prácticos que están pensados desde la perspectiva de la mejora y actualización de nuestras actividades y dispositivos formativos en el seno de cada titulación. Cambios que serán documentados y evaluados. Propuesta de cambio que se ha formalizado en un proyecto que constituye su guía de desarrollo y compromisos. 


\section{MODELOS DE INNOVACIÓN EN LA UNIVERSIDAD}

Afortunadamente, el panorama actual de las innovaciones que se están llevando a cabo en las universidades es muy amplio y diversificado. Muchas personas e instancias están vinculadas en proyectos de innovación de muy variada naturaleza y proyección. Por eso resulta pertinente buscar algún sistema de categorización. En ese sentido, hay muchas posibilidades de clasificación de las innovaciones. Las más habituales son las que las organizan:

a) por tipos: según los contenidos a los que afecte la innovación (currículum, organización, relaciones interpersonales, etc.).

Un repaso somero a los contenidos de los proyectos de innovación que se presentan a las diversas convocatorias de las universidades deja bien a las claras que el contenido fundamental de las innovaciones suele tener que ver con la introducción de las TIC en la enseñanza. Hoy por hoy, las nuevas tecnologías constituyen el eje vertebrador del esfuerzo innovador en la docencia universitaria. Son muy minoritarias las iniciativas que están orientadas a la mejora de los métodos docentes o de los materiales que se les entregan a los estudiantes.

b) por modalidades: según las diversas formas de llevarlas a cabo : individuales, grupales, de centro, etc. De origen externo, interno. Prescritas, guiadas, autogeneradas, etc.

Aunque la teoría didáctica ha dejado claro que la capacidad de impacto de las innovaciones está condicionada a la ruptura del individualismo (innovaciones en el seno de una clase son más fáciles de desarrollar pero poseen un impacto mínimo sobre la formación de los sujetos), la mayor parte de las innovaciones se mueven en ese ámbito. Esta circunstancia ha llevado a algunas universidades a imponer como condición o criterio de selección de las innovaciones el que éstas tuvieran que estar subscritas por más de un profesor: propuestas interdisciplinares, actuaciones transversales con varias materias implicadas, etc.

c) por niveles de impacto: según los ámbitos a los que afecta la innovación propuesta.

El nivel de impacto de una innovación puede variar mucho según el contenido de la innovación, los destinatarios de la misma y el tipo de instancias institucionales implicadas. En el punto siguiente analizamos estos niveles de impacto.

Niveles de impacto de la innovación.

Se ha considerado tradicionalmente que la unidad docentes en la universidad es cada profesor individual. Se supone que cada uno de nosotros hemos de organizarnos para desarrollar nuestra actividad formativa de forma autónoma y discrecional (cada uno ha de buscarse la vida por su cuenta y montárselo lo mejor que sea capaz). De ahí que la cultura individualista impregna todos los resquicios de la docencia y resulta muy difícil salir de ahí. Esta característica de la vida universitaria afecta de una manera clara a los formatos y modalidades de innovación en la universidad. La única posibilidad de que las estructuras y las prácticas universitarias se transformen es que se coaliguen en dicho proceso las diversas instancias y que se produzca una conjunción de sinergias de personas, recursos y oportunidades de mejora de forma 
tal que se genere nuevo conocimiento (una nueva mentalidad) y que de él se deriven nuevos procesos y nuevas prácticas. Por eso los estudiosos de la innovación acostumbran a diferenciar diferentes niveles y dimensiones en los procesos de innovación (Aurelio Villa, 2004)'.

ELMORE$^{2}$, especialista en el análisis de innovaciones educativas señala que en la actualidad existen tres grandes "orientaciones" desde las que se está planteando la innovación:

a) modelos de innovación basados en la reforma de la tecnología de la enseñanza.

Son los más frecuentes, si entendemos el concepto de tecnología en su sentido más amplio: todos los dispositivos puestos en marcha para propiciar el aprendizaje de los estudiantes. En ese sentido podríamos englobar aquí cualquier innovación dirigida a introducir modificaciones en cuanto a los modos de planificación, las metodologías, los recursos de instrucción, las TIC, la evaluación, etc. Obviamente este tipo de innovaciones puede ser llevado a cabo según modalidades muy diferentes: como experiencias individuales, como experiencias institucionales, surgiendo de la presión burocrática o siendo generadas en el interior del centro escolar, etc. Este tipo de innovaciones tiene un impacto fundamentalmente interno (referido a la instancia donde se lleva a cabo y condicionado a la amplitud de los participantes y al nivel de institucionalización logrado) y por lo general son capaces de modificar las prácticas pero no los modelos.

b) Cambios basados en la mejora de las condiciones ocupacionales de los profesores y profesionales de la educación.

En esta orientación es donde podemos incluir las innovaciones que afectan a cambios organizativos (modificación de horarios, estructuras curriculares modulares, agrupación o separación de centros o titulaciones, sistemas de trabajo en grupo, etc.). Entrarían también las experiencias de incentivación y refuerzo de la carrera profesional (aparición de nuevas funciones profesionales, redistribución de competencias en el funcionamiento de las instituciones o en la gestión del currículo, sistemas de acreditación de méritos, estímulos a la innovación, etc.); las variaciones salariales (aparición de nuevos complementos, reducción de horarios, etc.) y los programas de formación del profesorado.

Este segundo tipo de innovaciones tiene una incidencia directa sobre la organización, pero sólo una repercusión indirecta y difícil de establecer sobre la docencia concreta (lo que sucede dentro de las clases y los laboratorios). Este tipo de cambios afectan, con frecuencia, más a la reducción de las causas de descontento que al aumento de la motivación y compromiso personal de los profesionales. No es infrecuente que surjan conflictos de intereses entre los diferentes colectivos de la comunidad educativa (lo que supone una ventaja para unos no siempre tiene el mismo

1. Villa, A. (2004) : "Evidencias de Innovación en el Sistema Universitario". Ponencia presentada al III Symposium Iberoamericano de Docencia Universitaria. Univ. de Deusto (Bilbao), 21-24 de Enero 2004.

2. Elmore, R.F. et alii (1990) : Restructuring Schools : the next generation of Educational Reform. Jossey-Bass Publ. Oxford. 
cariz positivo para los otros: las ventajas de horarios para los profesores a veces conlleva empeoramientos en el de los estudiantes).

c) Cambios referidos a las relaciones entre las instituciones formadoras y sus clientes.

En este tercer apartado de innovaciones podríamos introducir toda una serie de modificaciones producidas en las universidades en los últimos años. Por ejemplo, las nuevas modalidades de captación y acceso de los alumnos al proceso formativo; la modificación de los contenidos formativos en función de las demandas externas y los nuevos perfiles profesionales; la incorporación de otros agentes formativos al desarrollo de los programas de formación (prácticum, intercambios con otras universidades, reconocimiento de competencias adquiridas fuera de la universidad, etc.); el establecimiento de nuevos dispositivos y modalidades de apoyo, guía y supervisión de los estudiantes (materias de nivel 0; tutorías de diverso signo a lo largo de la carrera; establecimiento de proyectos de estimulación de sistemas de autoempleo al final de las carreras).

En cuanto a las consideraciones generales que se pueden hacer con respecto a las innovaciones incluidas en esta tercera orientación se podría decir que son cambios que tienen incidencia directa en los aspectos organizativos y en la distribución de competencias. Sus influencias sólo son indirectas en el resto de las dimensiones escolares (mejora de las clases, el aprendizaje efectivo de los alumnos). Han ejercido un gran impacto en la superación de un concepto burocrático y rígido de la universidad y el currículo formativo que en ella se lleva a cabo: sobre todo las iniciativas dirigidas a conectar la formación de dentro con la formación fuera de la universidad han dado un nuevo cariz a toda la concepción tradicional de la enseñanza. Probablemente, sus efectos sobre la calidad de la enseñanza, cuando Ilegan a producirse (ya señalaba que su incidencia en la calidad de las prácticas formativas es indirecta) es más a largo plazo.

En un trabajo anterior, yo mismo señalaba que uno de los principales problemas de las innovaciones en la formación es el escaso nivel de impacto que alcanzan. Después de muchos años de convocatorias para proyectos de innovación, después del mucho esfuerzo realizado por instituciones y personas, no parece que podamos sentirnos satisfechos por los cambios reales logrados en la docencia universitaria. Estoy convencido de que esa falta de efectividad transformadora por parte de la innovación se ha debido a que, en buena parte, muchas de las innovaciones se han quedado en meras alteraciones de herramientas docentes (cambios en la tecnología) sin modificaciones profundas ni de los procesos ni en la dinámica del aprendizaje.

Es por eso que conviene insistir en que, desde el punto de vista del impacto de las innovaciones, es necesario que la innovación se produzca en un espacio poliédrico que tome en consideración todos sus componentes y dimensiones: doctrinales, personales, organizativos y culturales. En la innovación están implicadas personas (los profesores y profesoras que la llevan a cabo y los alumnos y alumnas sobre los que se proyecta). Pero resulta igualmente claro que no hay innovación (salvo la muy restringida) sin participación del contexto organizativo. De la misma manera que es claro, que lo organizativo formal deja de poseer capacidad de influencia (salvo en aspectos superficiales) a menos de que no venga acompañada de una "cul- 
tura" propicia a los cambios (por eso hay ciertas instituciones más dinámicas y con una mayor apertura y disponibilidad a incorporar cambios).

En la práctica, las innovaciones que funcionan bien suelen pasar con rapidez de la adscripción y dependencia personal a la adscripción institucional : es decir pasa de ser una cosa que afecta sólo al profesor que la promueve a ser algo que afecta y se ve afectado por la organización del centro. Cualquier tipo de iniciativa por simple y restringida que sea nos conduce enseguida a aspectos de tipo organizativo : horarios, uso de recursos institucionales, presupuesto, necesidad de tiempo, coordinación con otras actividades en marcha, etc. Es decir que, siendo cierto que no hay innovaciones sin profesores innovadores y que, por tanto, los profesores son las piezas clave de cualquier innovación, no es menos claro que tampoco hay innovaciones (salvo algunas muy restringidas y que van "de incógnito") si no existen condiciones organizativas que las hagan posibles. Y en cuanto entramos en cuestiones organizativas, la implicación de la institución en su conjunto parece necesaria.

Otro tanto se puede decir de la dimensión cultural: es difícil que prosperen procesos de innovación y mejora de las instituciones escolares si no se produce como condición previa una cierta (previsiblemente difuminada e incierta en sus primeras épocas) "cultura" preocupada por la calidad de la enseñanza y favorable a introducir las modificaciones necesarias para alcanzarla. Una cultura institucional básicamente conservadora e inmovilista provoca irremediablemente la asfixia de cualquier esfuerzo innovador (salvo que los individuos se lo planteen como una acción de francotirador y hasta "donde aguante el cuerpo").

Todo eso significa que innovar es una posibilidad cuyo pronóstico depende de que se produzca la conjunción positiva de varios factores : una idea que mejoraría las cosas, unas personas dispuestas a llevarla a cabo, unas condiciones institucionales que faciliten su desarrollo y que la apoyen.

También hemos de insistir en que las buenas innovaciones deben provocar un triple nivel de cambios: cambios en las cosas, cambios en las personas y cambios en la institución. El cambio en las cosas y su sentido es importante pero insuficiente como ha quedado claro, eso espero, en los puntos anteriores. Las innovaciones lo son porque incorporan nuevas modalidades de pensamiento y de acción, nuevos recursos, nuevas estructuras organizativas, etc. Pero con todo, eso no sería suficiente si a la vez no se produjera también cambio en las personas. Y en este caso no se trata específicamente de cambios que se hayan de producir en los alumnos sino más bien en los profesores y los otros agentes de la formación que participen en el proceso innovador (equipos rectorales, directivos de Facultades y Departamentos, profesores, alumnos, etc.). En la evaluación de la innovación habrá de tenerse en cuenta este aspecto de una manera muy especial. La pregunta a hacerse no se reduce a preguntarse ¿qué cosas han cambiado?. Otras preguntas deben ampliar ese sentido amplio de la innovación: ¿qué hemos aprendido nosotros de todo este proceso?, ¿en qué hemos cambiado nuestras ideas sobre la enseñanza, o sobre nuestra disciplina, o sobre los alumnos?, ¿en qué ha cambiado nuestra Facultad o Escuela al socaire de este proyecto de innovación?. La cuestión básica de las innovaciones no se culmina con el hecho de "hacer cosas distintas", hay que llegar a "pensar de forma distinta": valorar de forma distinta lo que hemos de hacer, ampliar nuestras perspectivas profesionales, nuestro conocimiento de los procesos, nuestras actitudes, etc. En general, poco bueno se podría decir de un proceso de innovación del cual salieran 
sus protagonistas en las mismas condiciones en las que entraron (como si la innovación hubiera pasado por ellos "sin romperlos ni mancharlos").

En esta misma línea de identificar y tomar en consideración el nivel de impacto de las innovaciones, Eckel y Kezar $(2003)^{3}$ proponen un modelo de análisis de las innovaciones en función de dos dimensiones: profundidad y extensión de las innovaciones. Las innovaciones se van distribuyendo así en los espacios intermedios de dichos ejes:

a) Innovaciones con una profundidad baja y una extensión alta son innovaciones de tipo epitelial. Son cambios de poca intensidad pero que afectan a muchos profesores: todos los profesores han de hacer sus programas y presentárselos a sus alumnos a comienzo de curso.

b) Innovaciones con una profundidad baja y una extensión también baja dan lugar a ajustes, esto es pequeños cambios parciales: en el horario, en la incorporación de retroproyectores a algunas materias, etc.

c) Innovaciones con una extensión baja pero una profundidad alta dan lugar a cambios aislados: cambios en las formas de evaluación y de calificación.

d) Innovaciones con una profundidad alta y una extensión alta dan lugar a cambios transformacionales, las innovaciones por antonomasia porque logran reconstruir los procesos en su conjunto. A este tipo de cambios nos podría llevar, por ejemplo, la incorporación de las competencias al diseño curricular.

Ni qué decir tiene que las innovaciones de mayor interés son éstas últimas por la capacidad de mejora profunda que poseen.

\section{LA FORMACIÓN DEL PROFESORADO UNIVERSITARIO}

Concluyamos de lo visto en el apartado anterior que ninguna innovación es viable sin un programa paralelo de formación del profesorado. Para algunos profesores este tipo de afirmaciones resultan chocantes e, incluso, ofensivas. Es como si se pretendiera decir que ellos no son capaces de innovar sin tener que recibir cursos para ello. Después de tantos años de experiencia como profesores les parece más que demostrada su capacidad docente e innovadora. Pudiera ser que tuvieran razón, pero más como excepción que como regla general. Lo más plausible es considerar que si se han mantenido las metodologías y estrategias didácticas tan ancladas en los sistemas tradicionales es porque los profesores no dominamos otras herramientas. Por eso se hace precisa la formación. Con los muchos años de experiencia docente que muchos de nosotros poseemos, bastaría un poco más de esfuerzo formativo (conocer y dominar otras formas de enseñanza) para que las clases se transformaran. Porque, en todo caso, a innovar también se aprende.

En ese sentido ha planteado Lewis Elton $(1997)^{4}$ que el principal objetivo en este momento es conseguir un nivel general de competencia docente que resulte cohe-

3. Eckel, P.D. y Kezal, A (2003): Taking the reins. Institutional transformation in Higher Education. Westport: Preager Pub.

4. Elton, L. (1997) : "Criteria for Teaching Competence and Teaching Excellence in Higher Education", en AYLETT, R. y GREGORY, K. (Ed.): Evaluating teacher quality in Hingher Education. London: Falmer Press. Pags. 33-42. 
rente con el propósito de la mejora de la docencia. No hay que proponerse objetivos desmesurados, al menos como punto de partida. Lo fundamental debe ser el posibilitar que el profesorado incompetente pase a ser competente y que los competentes gocen de condiciones propicias para desarrollar iniciativas de mejora.

Elton diferencia entre competencia docente y excelencia docente. La primera es fundamental y debería ser exigida a todo profesor universitario. La segunda expresa una especial cualificación docente y debería ser valorada como criterio de promoción docente en el mismo plano que la excelencia investigadora.

No es fácil establecer qué sea un docente competente ni cuáles son las supuestas competencias que debe poseer un docente, ni cómo se llega a obtenerlas, sobre todo en un contexto como el nuestro en el que no existe exigencia previa alguna (relacionada con la docencia) para iniciarse como docente universitario.

En un trabajo reciente ${ }^{5}$, he tratado de explicitar las 10 competencias que, a mi modo de ver, caracterizan lo que un docente universitario debe poseer para desarrollar "con competencia" su trabajo formativo. Tienen que ver con Planificar el proceso de enseñanza-aprendizaje; Seleccionar y presentar los contenidos disciplinares; Ofrecer informaciones y explicaciones comprensibles; Manejar didácticamente las Nuevas Tecnologías; Gestionar las metodologías de trabajo y las tareas de aprendizaje; Relacionarse con los alumnos; Tutorizar a los alumnos; Evaluar; Reflexionar e investigar sobre la enseñanza; Implicarse con la institución. Cada una de dichas competencias sirven, a su vez, como referentes para poder analizar la calidad de nuestra docencia.

También Elton, en el trabajo antes citado, explicita algunas de esas competencias: la de Organización (planificar, preparar, gestionar el tiempo, alcanzar los objetivos); Presentación (claridad, conocimiento de los temas, habilidades de explicación); Relaciones Interpersonales (entusiasmo por lo que enseña; empatía con los estudiantes, implicación de los estudiantes, sentido del humor); Orientación (adaptar los objetivos, estimular el aprendizaje); Evaluación (autorreflexión, sensibilidad por el feed-back, evaluación entre iguales).

En todo caso no se trata de establecer grandes listados de condiciones o cualidades. No suelen servir para nada. Lo importante es dejar claro que la buena enseñanza no se sustancia con una buena explicación en clase. Ser un buen explicador es algo estupendo y ayuda a que los estudiantes aprendan, pero ser un buen docente implica otros muchos elementos. Pasar de la competencia docente a la excelencia amplía aún más el abanico de espacios que los docentes universitarios deberíamos dominar y en función de los cuales habría de producirse nuestra progresión profesional.

En el trabajo antes citado, Elton especifica algunos de esos posibles criterios de excelencia en los docentes universitarios: poseer un alto nivel en las diversas competencias antes mencionadas; ser un docente que "reflexiona sobre su práctica"; ser un

5. Zabalza, M.A. (2003) : Competencias docentes del profesorado universitario. Madrid: Narcea. 
profesor/a comprometido con la innovación didáctica; estar en condiciones de diseñar propuestas curriculares y de organizar cursos de formación; desarrollar investigaciones que tengan que ver con la docencia en su propio ámbito de especialidad; dirigir o coordinar grupos docentes; etc.

Como podrán constatar muchas de estas líneas de mejora como profesionales son las que se trata de alimentar a través los programas de formación. Aquella vieja idea de que el buen profesor sabe su materia y sabe explicarla se ha quedado estrecha. El buen profesor y la buena profesora universitaria tienen ante sí en la actualidad un conjunto de retos mucho más amplios. Hoy en día, decir que enseñar en la universidad resulta una tarea compleja parece obvio. Quizás sea ése el punto de partida necesario para poder entrar a considerar qué tipo de preparación precisamos los profesores para poder afrontarla con éxito. En no pocas ocasiones la enseñanza se ha convertido en algo repetitivo que deja insatisfechos tanto a los profesores como a los alumnos. La idea de que dedicar mucho tiempo a la docencia supone restar tiempo a las otras tareas en las que solemos estar implicados y que con frecuencia nos abruman (reuniones, investigaciones, preparación de publicaciones, asistencia a congresos, etc.) ronda inevitablemente nuestra cabeza como una mosca cojonera. Pero a la vez, estoy por asegurar que somos muchos los que sentimos una mezcla de frustración y de sentimiento de culpa por el poco tiempo que dedicamos a preparar las clases, a supervisar el trabajo de nuestros alumnos, a atenderlos en sus demandas. Demasiadas ocupaciones, demasiados frentes abiertos. Resulta imposible atenderlos todos con eficacia. Sólo si la docencia acaba por constituir, en algún momento de nuestra vida, una prioridad será posible centrar en ella nuestro esfuerzo y nuestro tiempo lo suficiente como para equiparnos con el abanico de competencias al que antes he aludido.

Una situación parecida se tiene en relación a la innovación. Resulta difícil estar abierto a innovaciones cuando éstas van a exigir más trabajo y mayor atención a la docencia. En una agenda tan sobrecargada como la de los docentes universitarios actuales parece poco previsible cualquier tipo de cambio que altere las actuales coordenadas de dedicación y esfuerzo. Y no siempre por falta de motivación sino por falta de condiciones o por dificultades personales importantes para alterar la estructura de prioridades en las que nos movemos (lo que significaría pasar a primer lugar la docencia haciendo descender en el escalafón otro tipo de demandas: conferencias, cursos, asesorías, investigaciones, reuniones, etc.)

De ahí que la prioridad tanto en lo que se refiere a la innovación como a la formación para la docencia suela estar dirigida al profesorado novel. Son ellos y ellas quienes llegan a la universidad con una mayor carga de ilusiones y energías. Y menos abrumados de compromisos. Lástima que pronto se socializan en la idea de que para progresar en la carrera académica lo importante es investigar y que nadie va a entrar a considerar si su docencia es de calidad o no.

Las evaluaciones sobre el impacto de la formación para la docencia ofrecen datos muy esperanzadores. Incorporarse a algún programa de formación es la mejor garantía de que se retornará a otros. Son los profesores y profesoras que han asistido a algún programa de formación los que más demandan poder continuar formándose, quienes sienten más la necesidad de profundizar y de abrir nuevos frentes de mejora en su docencia. Se diría que lo importante es descubrir que la enseñanza es una tarea a la 
vez compleja y apasionante. Y que uno puede mejorar como docente a medida que va ampliando su conocimiento y enriqueciendo su repertorio de recursos didácticos. Por el contrario, son los que nunca asistieron a ninguna experiencia formativa los que menos necesidad sienten de ella y los que menos las demandan cuando se ofrecen en sus universidades.

\section{La formación del profesorado universitario: retos y posibilidades.}

La necesidad de la formación docente en la universidad, su sentido, sus contenidos, sus formatos y condiciones organizativas, etc. han quedado ampliamente analizadas en un texto anterior ${ }^{6}$. No insistiré aquí en ello. Me gustaría referirme, eso sí, a algunos de los problemas o condiciones que caracterizan a la innovación en la docencia universitaria y cómo eso afecta a la formación del profesorado. En ese sentido, yo diría que algunos de los principales problemas que la formación debe afrontar son los siguientes:

1) El problema de la mentalidad o las ideas imperantes sobre la innovación docente y sobre la propia formación para poder llevarla a cabo.

La formación suele carecer de sentido y de impacto real cuando es algo "forzado". Ése es uno de los dramas que deben afrontar los programas formativos (tanto en lo que se refiere a nuestros alumnos como en lo que nos afecta a nosotros como profesores). Si no sentimos la "necesidad" de la formación, ésta carecerá de sentido. Quizás participemos en ella, si nos obligan a hacerlo, pero nos implicaremos poco en ella y su valor para nosotros quedará muy mermado.

En estos últimos meses de trabajo sobre el proceso de convergencia europeo me he encontrado con muchos profesores que expresaban, por cierto con mucha seguridad y aplomo, que ellos no veían por qué se habían de cambiar las cosas si tal como estaban funcionaban bien. Para alguno de ellos siempre se ha hecho así y, además, no hay otra forma de hacerlo. Es difícil suponer que en ese contexto actitudinal vayan a estar disponibles y abiertos a iniciativas innovadoras.

Por eso el tema de la mentalidad resulta crucial en la formación de los docentes. El primer paso de la estrategia ha de ser, justamente, destacar el valor de la docencia y la importancia de la formación para conseguir su mejora. Pero esta modificación de la mentalidad no suele producirse de forma espontánea. Un querido profesor de Didáctica nos explicaba en sus clases que para que nuestros alumnos aprendan son precisos tres tipos de procesos docentes que se complementan entre sí: la evitación (debemos eliminar todos aquellos obstáculos que impidan el aprendizaje y ofrecerles los recursos necesarios para que el aprendizaje se produzca); la seducción (debemos hacer atractivo el aprendizaje a nuestros alumnos, motivarlos a aprender) y, finalmente, la presión (utilizando los mecanismos apropiados para que, si los otros procesos no funcionan adecuadamente, los alumnos se vean forzados a esforzarse y cumplir las exigencias marcadas). Algo similar podría decirse de la formación. Las Universidades precisan de sistemas que combinen mecanismos y condiciones de posibilitación, seducción y presión que propicien la progresiva incorporación de sus académicos a procesos de formación. Se han de eliminar los obstáculos (sobrecarga horaria, falta de tiempo, falta de oferta formativa, etc.), se ha de hacer atractiva la formación (reconociéndola como mérito, asegurando su calidad, adecuándola a las necesidades y los 
tiempos disponibles, etc.) y, si fuera necesario, se han de emplear mecanismos de presión directa o indirecta que ayuden a superar las reticencias o desinterés (exigiendo ciertos niveles de acreditación como docentes, vinculándola a la evaluación, condicionando la promoción o la mejora salarial a la participación en programas de formación, etc.).

\section{2) La disponibilidad de tiempo.}

Uno de los principales problemas del profesorado universitario actual es el de la escasa disponibilidad de tiempo. Pese a la existencia de un cierto estereotipo social que insiste en que los profesores trabajamos poco y tenemos muchas vacaciones, la verdad es que somos, en general, personas excesivamente ocupadas. En algunos casos en compromisos intra-universitarios (reuniones, investigaciones, informes, atención a la docencia, participación en comisiones o grupos de trabajo de diverso signo, etc.). En otros casos, en compromisos externos a la universidad aunque vinculados a la función de "extensión" universitaria: cursos, conferencias, informes, asesorías, etc. Para algunos colectivos docentes el problema del tiempo adquiere aún tintes más dramáticos: profesores contratados por horas; profesores asociados que combinan su propio trabajo con la impartición de algunas clases en la universidad; profesores que han de trabajar en distintas universidades o en distintos centros de enseñanza, etc.

Y, sin embargo, la formación, para ser eficaz, exige tiempo, dedicación, constancia y esfuerzo. Si no se dispone del tiempo suficiente es difícil que la formación funcione. Aparece como una carga más a la que los docentes debemos atender, con lo cual, al final, hasta puede tener efectos contraproducentes (más estrés, menos tiempo disponible para atender adecuadamente la docencia, etc.).

A veces, para obviar este problema se buscan formatos ligeros y cortos: cursos de pocas horas, congresos, etc. Pero eso sirve poco. No basta con recibir dosis concentradas de información. La formación requiere de tiempo de sedimentación de las informaciones, de contraste práctico (poder aplicar lo que se ha estado trabajando) y debate posterior, etc. De ahí la importancia estratégica que están teniendo los programas de formación semipresenciales que consumen menos tiempo y permiten al profesorado adecuar su dedicación a sus propias condiciones de vida. Pero, a su vez, este tipo de programas, debilita la parte social de la formación. Por lo general, al menos en lo que se refiere a procesos de actuación didáctica, uno no aprende a solas. No basta con leer un libro o desarrollar una actividad propuesta por Internet. Se aprende discutiendo con los colegas, contrastando e intercambiando experiencias, compartiendo soluciones y recursos. Incluso en programas semipresenciales se ha de garantizar este "aprendizaje coral" de que hablaba Vigotsky.

3) El problema de la ausencia de referentes y ejemplos de buenas prácticas.

Una de las consecuencias de la "opacidad" de lo que sucede en las clases es que los profesores carecemos, por lo general, de referentes. Como no sabemos lo que hacen los otros en sus clases, tenemos escasas posibilidades de contrastar nuestra experiencia con la suya.

Los referentes son fundamentales para convertir las ideas abstractas sobre la calidad de la docencia (lo que se debe hacer) en condiciones y actuaciones específicas. La literatura pedagógica los ha denominado a veces ejemplificaciones porque sirven justamente como ejemplos prácticos y situacionales de lo que se puede hacer en la didáctica universitaria. En la actualidad, los procedimientos del benchmarking (identificación de buenas prácticas que sirven de punto de referencia para nuestras propias evaluaciones) están teniendo una amplia acogida como sistema para establecer prioridades y estrategias de mejora institucional. 
No es fácil atravesar los muros del aula. Pesa mucho la tendencia al individualismo y a un cierto "ocultismo" de lo que hacemos en nuestras clases (¿quién sabe si no será consecuencia de una cierta inseguridad en lo que hacemos?). Resulta muy trabajoso conseguir permiso para observar clases o para grabarlas con fines de aprendizaje. Y, sin embargo, una de las condiciones de la calidad es justamente la de la visibilidad. La visibilidad hace posible que conozcamos lo que hacen nuestros colegas y nos podamos enriquecer del contraste entre nuestras experiencia y las suyas. Por otra parte, la visibilidad actúa también como fuente de presión sobre nosotros mismos: cuando uno sabe que lo que hace va a ser visto y analizado por otros se esfuerza más. Ésa ha sido una de las consecuencias más notables de la incorporación de las nuevas tecnologías a los procesos de enseñanza universitarias. Cuando todo quedaba en la intimidad del trabajo en el aula y los intercambios entre profesor y alumnos, la cosa era sencilla. Pero ahora, somos conscientes de que los programas que ofrecemos, los materiales que ponemos a disposición de nuestros alumnos, las evaluaciones que les hacemos, etc. pueden a ser vistas por otros, y eso nos lleva a tentarnos más los machos y a cuidar más lo que hacemos.

Ser un buen docente no tiene que significar que uno tenga que "crear ex novo" todo lo que hace en sus clases. Sería absurdo, aparte de agotador e inviable. Por eso resulta tan interesante poder contar con las aportaciones y ejemplos de otros colegas. Está teniendo mucho éxito y espero que la iniciativa se extienda mucho más en los próximos años la creación de "Bases de recursos compartidos" entre universidades y profesores. Profesores que estamos impartiendo disciplinas similares en diversas universidades podemos fácilmente disponer de un sitio en Internet en el que "colguemos" los materiales que utilizamos en nuestras clases, desde el programa hasta las prácticas programadas, desde los dossieres ofrecidos a los alumnos hasta las evaluaciones.

Ése es, también, el propósito que las asociaciones como $\mathrm{AIDU}^{7}$ tratamos de hacer posible: generar espacios de debate e intercambio de experiencias entre académicos iberoamericanos que nos permitan enriquecernos mutuamente.

\section{4) La necesidad de estrategias de formación adecuadas.}

Está fuera de toda duda que el profesorado universitario precisa de un estilo de formación bastante peculiar, que no siempre coincide con el empleado con profesores de otros niveles educativos. La gente de Universidad posee características muy diversas y pertenece a tradiciones profesionales muy particulares. Sus problemas docentes no son muy diferentes (al final, el problema es cómo potenciar el aprendizaje de los alumnos, como organizar la docencia, cómo seleccionar buenos contenidos, cómo evaluar, etc.) pero sí lo es su forma de abordarlos. Una de las condiciones particulares que debe atender la formación del profesorado universitario tiene que ver con el elevado nivel intelectual que, en principio, se nos supone a los profesores universitarios (se le supone a todo el profesorado, al margen de cuál sea el nivel en que enseña, pero es en la Universidad donde se diría que el profesorado lo vive más como "marca de identi$\mathrm{dad}^{\prime \prime}$ ). Por eso, suelen aceptar mal enfoques excesivamente practicones y centrados en actuaciones muy concretas y casuísticas. Pero como, a la vez, son profesionales com-

7. AIDU (Asociación Ibedroamericana de Didáctica Universitaria) tiene su sede, en la actualidad, en Santiago de Compostela donde nació en 1999. Propicia intercambios entre docentes iberoamericanos de las diversas áreas de conocimiento, convoca un Congreso bianual (alternativamente en América y en Europa) y facilita el establecimiento de redes entre universidades iberoamericanas (para desarrollar proyectos de investigación, innovaciones, materiales didácticos, etc.). Pueden contactarnos por e-mail: zabalza@usc.es; dofetria@us 
prometidos con la práctica tampoco aceptan discursos abstractos y que se alejen en exceso de lo que es la actuación didáctica. Alguien lo ha llamado la "ética de la practicidad".

En los últimos años se han ido poniendo en marcha en las universidades proyectos muy interesantes de formación de su profesorado. No todos ellos han dado los resultados que se esperaban, pero en general se puede afirmar que han supuesto un sustancial aporte al cambio de mentalidad al que he aludido antes y al equipamiento conceptual y técnico del profesorado que ha participado en ellos (todavía, salvo excepciones, muy minoritario). Entre los modelos que mejor han funcionado podríamos destacar los siguientes:

- Los modelos basados en el apoyo mutuo. Sin duda, los más eficaces y con mayor capacidad de impacto en la mejora de la docencia. A esta categoría perteneces los programas de senior teachers (profesores con experiencia que tutorizan a los noveles); programas que incluyen la colaboración en la formación por parte de aquellos que han ido pasando por programas de formación; programas basados en equipos de innovación; programas basados en la génesis de bases de recursos compartidas, etc.

- Los modelos basados en la participación en programas de investigación-acción. También en este caso se trata de iniciativas que poseen, por su vinculación a la investigación, un fuerte atractivo para los docentes universitarios. Ejercen, además, un alto nivel de impacto sobre la docencia. Grupos de profesores que participan en proyectos de mejora de su actuación concebidos como procesos de investigación didáctica aplicada. En algunos casos se trata de elaborar y experimentar materiales didácticos y/o guías para el aprendizaje de sus estudiantes. En otros casos, se somete a estudio sistemático algunas variables que afectan al aprendizaje y/o la performance académica de los estudiantes: errores conceptuales, motivación, estrategias de estudio o de resolución de problemas, estilos de aprendizaje, etc. Han sido numerosos, también, los grupos que se han vinculado a proyectos de innovación didáctica que se somete a estudio sistemático por parte de quienes participan en ella: procesos de trabajo interdisciplinar, sistemas de trabajo tutorizado, modelos de trabajo autónomo o a distancia, etc. En algunos casos (muy escasos en el contexto español, aunque comienzan a ser frecuentes en otros países) los sistemas de acreditación en docencia universitaria (maestrías o doctorado) incluyen la realización de investigaciones más formalizadas sobre cuestiones didácticas relacionadas con la disciplina que se imparte.

- Un tercer tipo de modelos de formación son los basados en el enriquecimiento doctrinal de los docentes. Como he señalado en un punto anterior, uno de los cambios que la formación precisa es el cambio de mentalidad sobre la enseñanza y su fundamentación. Enriquecer el conocimiento que los profesores tenemos sobre la enseñanza y el aprendizaje en el contexto universitario constituye una condición sine que non para que la calidad de la enseñanza progrese. Al final, ser un buen profesor (como ser un buen profesional en cualquiera de los campos) significa ser un especialista o, al menos, un buen conocedor de los procesos de enseñanza y aprendizaje. Ese es el papel que juegan los cursos y talleres sobre docencia universitaria hoy día presentes en la mayor parte de las universidades. También cabe incluir en esta categoría interesantes experiencias en la distribución entre el profesorado (o su oferta a través de la red) de fichas o dossieres sobre cuestiones relacionadas con la docencia (explicación de conceptos, exposición de experiencias, descripción de métodos, presentación de posibles materiales para la evaluación, la observación, etc.). El mismo sentido tiene la puesta a disposición del profesorado de bibliografía especializada en cuestiones de docencia universitaria.

- Finalmente, quisiera referirme al sistema en el que yo mismo he ido trabajando: los modelos basados en la documentación y la reflexión. Esta última década ha sido, en lo que se refiere a la formación de profesores, la época de la "reflexión". Se ha insistido en la necesidad de formar profesores "reflexivos" capaces de someter a análisis sus propias prácticas. No estoy seguro de que hayamos avanzado mucho en esa dirección, 
pero lo que sí ha quedado claro es que la práctica por sí misma no genera conocimiento ni mejora la calidad de lo que se está haciendo. Para eso se requiere una práctica revisada. Con ese propósito se han planteado procesos formativos basados, por ejemplo, en la grabación en vídeo de clases y su análisis posterior. Esa posibilidad ofrecen también los portafolios en la medida que constituyen una selección representativa de nuestro trabajo y nos permite analizarlo en perspectiva. Lo mismo sucede con los diarios de clase que permiten un trabajo muy profundo de auto-conocimiento y de revisión y mejora de las propias prácticas docentes ${ }^{8}$.

\section{CONCLUSIONES}

Para concluir, y a la vista de cómo están las cosas en la docencia universitaria, todo lo anterior se pudiera resumir en algunas consideraciones referidas a los nuevos caminos que se nos abren para la innovación en la universidad y, consecuentemente, para orientar la formación que el profesorado universitario precisa.

Debemos aprovechar la movida que se ha puesto en marcha con esta historia del proceso de convergencia hacia un Espacio Europeo de Educación Superior. Se trata de un proceso con mimbres suficientes como para hacer buenos cestos, pero que, al igual que nos ha pasado con otras oportunidades anteriores, pudiera quedarse en un puro maquillaje institucional. Si al final todo queda en que hemos reconvertido nuestros créditos convencionales (las 10 horas de docencia) en créditos ECTs., habrá sido el parto de los montes (un puro ajuste, en la terminología de Eckel y Kezar).

Muy al contrario, la propuesta de renovación de la docencia universitaria que nos llega de Europa trae consigo retos tan sustantivos como el cambio de eje de la docencia, pasándolo de la enseñanza al aprendizaje (the shift from teaching to learning); potenciar el aprendizaje autónomo pero guiado de los estudiantes; organizar la formación en base a las competencias (no sólo profesionales sino de formación global), etc.

Este conjunto de nuevos retos configura un nuevo escenario especialmente propicio para la innovación y el desarrollo de nuevas iniciativas por parte de los docentes. Y para que eso suceda requiere, igualmente, que desde las instituciones universitarias se propicie ese plus de motivación y esfuerzo que se nos exige, y la formación necesaria para que estemos en condiciones de dar una respuesta efectiva a dichos retos.

Yo destacaría de entre los muchos retos que se nos abren en relación a la innovación los dos siguientes:

a) Un primer compromiso en ese sentido ha de ser, sin duda, el de mejorar nuestro equipamiento didáctico, sobre todo en lo que se refiere a los métodos docentes. La enorme extensión de la lección magistral como método casi único depende más de la dificultad para poner en marcha otros sistemas alternativos que de la voluntad explícita de los docentes de mantenerlo a toda costa.

8. Zabalza, M.A. (2003): Los diarios de clase: un recurso para analizar cualitativamente los dilemas prácticos de los profesores. Madrid: Narcea. 
El estudio y la comparación entre diversos métodos de enseñanza y sus efectos en el aprendizaje han sido una de las constantes de la investigación pedagógica. Merece la pena documentarse a este respecto y analizar el conocimiento ya alcanzado en relación a la metodología didáctica más pertinente al propio ámbito científico al que uno pertenece.

En un reciente e interesante artículo, los profesores de la Univ. de Friburgo, Oser y Baeriswyl (2001) ${ }^{9}$, han acudido a la analogía de las coreografías para referirse a la plasticidad de la enseñanza. Hasta 12 coreografías diversas distinguen estos autores. Parten de la idea de que la buena enseñanza cumple dos funciones básicas. Por un lado, crea las condiciones para que puedan llevarse a cabo acciones concretas por parte de profesores y estudiantes. Es la parte visible de la coreografía (la estructura de la lección o actividades formativas, la organización de los espacios y los tiempos, los recursos, etc.). Pero además, crea las condiciones para que se puedan desarrollar las actividades interiores y no visibles del aprendizaje por parte de los alumnos (las operaciones mentales que se realizan en el proceso de aprendizaje al que denominan, el modelo base del aprendizaje). De esta manera, el mismo proceso de enseñanza puede llevarse a cabo con coreografías muy diversas que tendrán, a su vez, efectos bien diferentes sobre el aprendizaje de los estudiantes. La idea común es que los profesores necesitamos movilizar la actividad "interna" que desarrollan los aprendices a través de un conjunto de condiciones externas e internas (las coreografías) capaces de romper el equilibrio anterior y de provocar en ellos nuevas inquietudes.

Parte de esta renovación metodológica ha de estar orientada, naturalmente, a propiciar ese aprendizaje autónomo de los estudiantes. Un aprendizaje autónomo pero "guiado". Y eso requiere la elaboración de "buenos materiales" en forma de guías didácticas, dossieres, etc. Las TIC constituyen también una herramienta muy importante en este sentido.

b) Desarrollar la cultura de la documentación, evaluación y reajuste de nuestras prácticas docentes. Ninguna mejora es posible sin esta condición. Uno puede llevar en la universidad muchos años y haber acumulado experiencia sin límite en su docencia, pero si no ha sometido a análisis su actuación, si no ha vuelto periódicamente sobre sus convicciones y sus prácticas docentes es bastante probable que se haya enrocado en viejas seguridades.

Curiosamente, es casi seguro que tal cosa resulta imposible en nuestros otros ámbitos de actuación: la investigación, la gestión, la extensión cultural, las colaboraciones profesionales, etc. Resultaría impensable mantenerse en el mismo círculo de conceptos, metodologías y orientaciones de hace 10-15 años. Pero sin embargo, casi no choca que uno siga enseñando de forma parecida a como lo hacía cuando comenzó o a como lo hacían nuestros propios profesores cuando éramos estudiantes. Se diría que la enseñanza, la buena enseñanza, se plantea y desarrolla como una actividad casi inmutable. Que no se ve afectada por nuevos estudios y avances en su organización y en los recursos aplicables. Nada menos cierto, desde luego.

9. Oser, F.K y Baeriswyl, F.J. (2001): "Choreographies of Teaching: Bridging Instruction to Learning", en RICHARDSON, V. (Edit.) : Handbook of Research on Teaching, Fourth Edition. Washington: AERA. Pags. 1031-1065. 
"Este libro asume que los mejores profesores son aquellos que saben por qué hacen lo que hacen cuando enseñan. Ellos toman en consideración diversas alternativas, sopesan sus posibles efectos y evalúan sus decisiones recogiendo evidencias relevantes", con esta idea como punto de partida comienza Studiyng Teaching, el libro de Raths, Pancella y Van Ness $(1971)^{10}$. Ése es también el objetivo básico en la formación del profesorado universitario. Hemos avanzado mucho en esa dirección, pero aún queda mucho camino por andar. Por eso resulta preciso seguir insistiendo en la necesidad de la formación y en que ésta es una tarea que nos incumbe a todos, desde los equipos rectorales a las Facultades, Departamentos, Equipos de investigación y profesores individuales. Sólo la sinergia en el esfuerzo colectivo hará que la calidad de la docencia mejore. 